\title{
La Perspective Judiciare sur la Réhabilitation des Drogues en Indonésie
}

\author{
Cecep Mustafa
}

Université de Stirling, France, E-mail: cecepmustafa97@gmail.com.

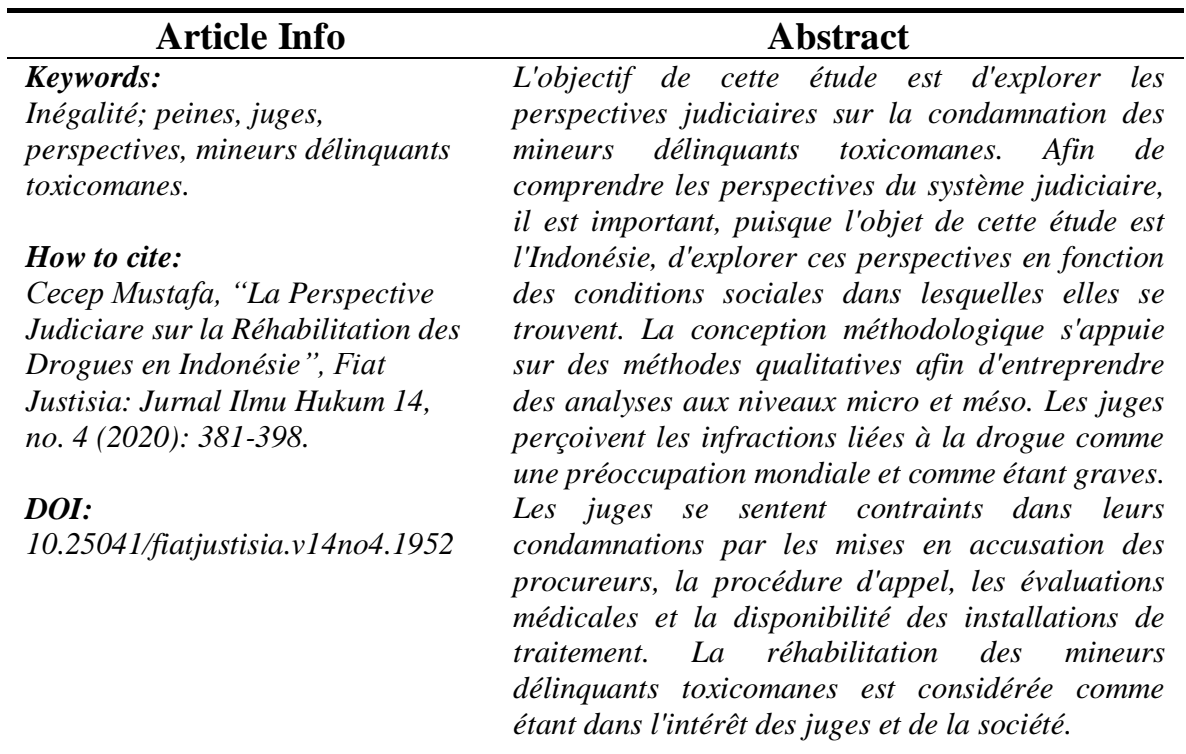

\section{A. Introduction}

Il est important de faire des recherches sur la perspective judiciaire de la réhabilitation des consommateurs de drogues. La réhabilitation des consommateurs de drogues semblerait représenter une rupture avec la recherche contemporaine sur le traitement des toxicomanies, qui a préconisé la nécessité d'un rétablissement des toxicomanes. ${ }^{1} \mathrm{Il}$ y a de plus en plus de preuves concernant les perspectives judiciaires en matière de condamnation. Il est important de comprendre les perspectives du pouvoir judiciaire pour des raisons théoriques. Les questions théoriques centrales sont de savoir si la détermination de la peine est une pratique sociale ancrée dans le contexte de la punition et de la société et si les perspectives judiciaires interagissent ou non avec la détermination de la peine. ${ }^{2}$ La compréhension de ces pratiques

\footnotetext{
1 J. Buchanan, "Ending Drug Prohibition with a Hangover", British Journal of Justice communautaire 13, no. 1 (2015): 55-74.

${ }^{2}$ Gibson, J.L. "From simplicity to complexity: The development of theory in the study of judicial behaviour", Political Behaviour 5, no. 1 (1983): 7-49. O. W. Holmes, The Common
} 
sociales de condamnation est également cruciale pour combler le vide dans la littérature sur la condamnation des mineurs délinquants toxicomanes. ${ }^{3}$

La recherche qui constitue la base de ce document offre un aperçu des tribunaux et des pratiques de condamnation contemporains en Indonésie ${ }^{4}$, ce qui peut éclairer à la fois les défis et les possibilités de réformer ces pratiques ${ }^{5}$. Afin de comprendre les pratiques en matière de condamnation, il est important, dans le cadre de cette est sur le cas de l'Indonésie, pour explorer les perspectives judiciaires dans les conditions sociales où ils opèrent ${ }^{6}$. Les recherches sur les attitudes et les perceptions des juges en matière de condamnation sont rares, et les difficultés d'autorisation et d'accès découragent les enquêteurs potentiels. Pour cette raison, il n'existe pratiquement aucun précédent pour ce type de recherche. Une étude similaire sur les peines prononcées par la Crown Court au Royaume-Uni a été tentée au début des années 1980, mais la permission a été refusée pour poursuivre audelà de l'étude pilote. ${ }^{7}$

Ce que les juges pensent de la détermination de la peine, et comment ils abordent cette tâche, peut être décrit comme une lacune dans la recherche sur la détermination de la peine. Le juge joue un rôle central dans le processus de détermination de la peine en raison du système international de discrétion judiciaire, et c'est à cause de cette discrétion limitée qu'il est important de savoir comment les juges prennent leurs décisions. Ce document se concentre sur la perception qu'ont les juges indonésiens de la condamnation des petits délinquants toxicomanes. Afin de comprendre les questions entourant ce processus, il est nécessaire d'examiner le contexte qui a conduit à l'application de la peine en matière de drogue en premier lieu.

Les prisons indonésiennes ont longtemps été confrontées aux problèmes liés à la consommation de drogue. La population carcérale a augmenté de 85

Law (USA: Harvard University Press, 2009). N. Hutton, "Sentencing as a Social Practice", dans S. Armstrong et L. McAra (eds.) Perspectives on Punishment: the Contours of Control (Oxford: Oxford University Press, 2006), 155-174. M. A. Myers, et S. M. Talarico, The Social Contexts of Criminal Sentencing (Springer Science et médias d'affaires, 1987)

${ }^{3}$ J. Ward, "Punishing Drug Possession in the Magistrates' Courts: Il est temps de Rethink", European Journal on Criminal Policy and Research 19, no. 4 (2013): 289-307. https://doi.org/10.1007/s10610-012-9191-1.

${ }^{4} \mathrm{~F}$. Vanhamme and K. Beyens, "La recherche end sentencing: un survol contextualize", Déviance et Société 31, no. 2 (2007): 199-228. https://doi.org/10.3917/ds.312.0199.

${ }^{5}$ Ashworth, A. Sentencing and Criminal Justice (Cambridge: Université de Cambridge Presse, 2010)

${ }^{6}$ Hutton, N. "Sentencing as a Social Practice", dans S. Armstrong et L. McAra (eds.) Perspectives on Punishment: the Contours of Control (Oxford: Oxford University Press, 2006), 155-174.

${ }^{7}$ Ashworth, A., Genders, E., Mansfield, G., Peay, J., et Player, E. Sentencing in the Crown Court (UK: Centre de recherche criminologique de l'Université d'Oxford, 1984) 
$\%$ entre 2011 et $2013^{8}$. La croissance de la population carcérale est en grande partie liée à la poursuite des délinquants toxicomanes. Selon le rapport 2013 de l'IPS, environ $93 \%$ des prisonniers dans tout le pays ont des antécédents de toxicomanie, dont $45 \%$ pour consommation de drogue, et $48 \%$ pour la vente de médicaments. ${ }^{9}$ La forte proportion de consommateurs de drogues en prison reflète les pratiques de ciblage et d'emprisonnement prolongé des consommateurs de drogues qui ont eu un impact sur le taux d'occupation des prisons de plus en plus surpeuplées. ${ }^{10}$

En raison de modifications récentes de la législation, la sanction légale, imposée pour les infractions graves liées à la drogue, y compris le trafic de drogue, sera plus longue (la période d'emprisonnement prévue par la loi sur les stupéfiants 35/2009 est d'un tiers plus longue que celle prévue par la loi sur les stupéfiants 22/1997) de l'emprisonnement jusqu'à la peine de mort. En ce qui concerne les infractions mineures liées à la drogue, y compris la possession et la consommation de drogue, la loi sur les stupéfiants (loi $35 / 2009$, règle 127) permet de choisir entre les infractions moins graves liées à la drogue, qui sont soit punies d'une peine d'emprisonnement, soit envoyées en traitement.

En 2014, il a été de plus en plus reconnu que la guerre contre la drogue avait conduit à l'emprisonnement d'un trop grand nombre de délinquants condamnés pour trafic de drogue. C'est dans ce contexte particulier que le règlement $\mathrm{n}^{\circ} 01 / 2014$ a été élaboré pour permettre une plus grande discrétion judiciaire. En même temps, le règlement offre plus de discrétion aux juges indonésiens pour développer des sanctions alternatives par lesquelles les délinquants, qui sont reconnus coupables et condamnés pour possession, contrôle et utilisation de drogues pour l'approvisionnement personnel, ne reçoivent pas de peines de prison, mais sont plutôt condamnés à un traitement. ${ }^{11}$ En bref, les changements dans le domaine pénal et en particulier le nombre croissant de consommateurs de drogues dans le système pénal ont façonné des conditions appropriées pour le recours à la condamnation pour la réhabilitation.

Cette étude présente les résultats d'une recherche primaire sur les perceptions des juges indonésiens en ce qui concerne la condamnation de mineurs délinquants toxicomanes, et a examiné les questions de recherche

\footnotetext{
${ }^{8}$ Service pénitentiaire indonésien. Rapport annuel (Jakarta: Ministère de la justice et des affaires humaines Rights) [en ligne] Disponible à l'adresse suivantehttp://www.ditjenpas.go.id/sites/default/files/ANUAL\%20REPORT\%20REVISI\%20 TE RAKHIR\%20hq\%20print.pdf [consulté le 8 février 2015].

${ }^{9}$ Ibid.

${ }^{10}$ Mulyadi Punition contre les trafiquants et les consommateurs de drogues : Research on Principles, Theory, and Practice Norms Application in Court (Jakarta: Centre de recherche pour la Cour suprême indonésienne, 2012)

${ }^{11}$ I. Iskander, "Decriminalisation to Drug Users are not similar with Legalisation". Disponible à l'adresse suivante : http://anangiskandar.wordpress.com/ [consulté le 6 octobre 2014].
} 
suivantes: Quelles sont les perspectives judiciaires concernant la condamnation des mineurs délinquants en matière de drogue en Indonésie?; Selon les juges, quels sont les facteurs qui les influencent lorsqu'ils condamnent des délinquants mineurs en matière de drogue devant les tribunaux indonésiens?; Quels sont les objectifs déclarés des juges des tribunaux indonésiens lorsqu'ils condamnent des mineurs les délinquants toxicomanes?

Cette recherches basées sur des entretiens ont fait appel à des juges indonésiens comme participants. Le présent document fournit quelques réflexions, en grande partie dans leurs propres mots, sur les problèmes auxquels les juges sont confrontés dans leur interaction avec le processus de condamnation. Dans ce document, j'aborde la réhabilitation des toxicomanes puisque c'est la question sur laquelle les juges ont eu les opinions les plus fortes.

L'histoire se répète car les personnes issues des classes inférieures ont été sévèrement condamnées, tandis que celles des classes supérieures n'ont souvent pas été sanctionnées pour des délits mineurs liés à la drogue. ${ }^{12}$ Des recherches critiques ont examiné les préjugés de classe et de race dans la criminalisation des délinquants noirs et de classe inférieure en matière de drogue, ${ }^{13} l^{\prime}$ emprisonnement de masse pour des délits mineurs liés à la drogue ${ }^{14}$ et les disparités dans les peines prononcées pour les infractions liées à la drogue. ${ }^{15}$ Les délinquants qui n'avaient pas les moyens de payer pour des programmes volontaires de traitement de la toxicomanie étaient plus

\footnotetext{
${ }^{12}$ W. J. Chambliss, "Toward a Political Economy of Crime", Theory and Society 2, no. 1: 149170. Melossi, D. Controlling Crime, Controlling Society: Thinking about Crime in Europe and America (Polity, 2008). R. Quinney, Critique de l'ordre juridique: Le contrôle du crime dans la société capitaliste (USA: Transaction Publishers, 1974). G. Rusche, et O. Kirchheimer, Punishment and Social Structure (USA:Transaction Éditeurs, 2003)

13 J. Buchanan, "Ending Drug Prohibition with a Hangover", British Journal of Justice communautaire 13, no. 1 (2015): 55-74. M. D. Lassiter, "Impossible Criminels: The Suburban Imperatives of America's War on Drugs", Journal of American History 102, no. 1 (2015): 126140. https://doi.org/10.1093/jahist/jav243. E. A. Nadelmann, "Criminologist and Punitive Drug Prohibition: to serve or to challenge? Criminologie et politique publique, 3 no. 3 (2004): 441-450. https://doi.org/10.1111/j.1745-9133.2004.tb00052.x. D. M. Province, "Race et inégalité dans la guerre contre la drogue", Revue annuelle du droit et de la politique de l'Union européenne Social Science 7 (2011): 41-60.

${ }^{14}$ B. Pettit, B. Western, "Mass imprisonment and the life course: Race and class inequality in US incarceration", American Sociological Review 69, no. 2 (2004): 151-169. https://doi.org/10.1177/000312240406900201. M. Shiner, "Drug policy reform and the reclassification of cannabis in England and Le Pays de Galles: A cautionary tale", International Journal of Drug Policy 26 (7), 696-704. https://doi.org/10.1016/j.drugpo.2015.03.009.

${ }^{15}$ E. Chen, et K. Nomura, "Et la justice pour tous ? Racial and Ethnic Disparities in Federal Drug Courts in California and the US', California Journal of Politics and Policy 7, no. 2 (2015). K. B. Nunn, "Race, crime and the pool of surplus criminality: or why the war on drugs was a war on blacks", Gender Race and Justice 6, no. 381 (2002). C. Spohn, "Evolution of Sentencing Research", Criminology and Public Policy 14, no. 2 (2015): 225-232.
} 
susceptibles d'être perçus par les juges comme étant moins aptes à la réhabilitation. Une étude récente sur le système judiciaire en Slovénie a indiqué que la loi sur les peines est conçue pour punir de manière disproportionnée les consommateurs de drogue. ${ }^{16}$

La panique morale concernant la consommation de drogues et la diabolisation de la consommation de drogues parmi les minorités dans les pays anglophones est souvent associée aux classes pauvres et aux minorités raciales. Qu'aux États-Unis (US), l'emprisonnement massif de personnes de couleur et d'immigrants pour des délits liés à la drogue peut être interprété comme une tentative de séparer les membres de la classe inférieure des membres de la classe moyenne. Les citoyens de la classe inférieure sont emprisonnés de manière punitive pour des périodes plus longues tandis que les citoyens de la classe moyenne restent cachés du système de justice pénale. Aux États-Unis, les lignes directrices en matière de condamnation pour les infractions liées à la drogue semblent être attribuables à une discrimination de classe et la sanction tend à être disproportionnée dans le cas des citoyens de la classe inférieure.

L'un des principaux défis est la difficulté de s'attaquer aux inégalités sociales, telles que la discrimination, les inégalités et les traumatismes de l'enfance, qui sont souvent devenus des facteurs de risque de la dépendance à la drogue. Il soutient qu'une combinaison de programmes de traitement de la toxicomanie et de sanctions pourrait avoir une incidence sur le comportement des toxicomanes. Les services de traitement de la toxicomanie devraient comprennent toute une série de personnes, telles que les jeunes, les parents d'usagers et les travailleurs du sexe qui consomment des drogues et subissent de graves conséquences pour eux-mêmes, leurs enfants et leurs familles. La mise en œuvre du traitement de la toxicomanie pose certains problèmes: les difficultés rencontrées par les femmes qui cherchent un traitement communautaire local, les lacunes dans les capacités de prestation de services, le manque de communication entre les prestataires, l'absence de philosophie de la part des prestataires, qui est source de confusion dans la communauté, et le manque de coordination entre les prestataires semblent être des obstacles. ${ }^{17}$

Un traitement réussi comme étant celui où les participants rapportent une expérience positive du traitement, où le personnel est disponible en cas de besoin et motive les clients/utilisateurs de services à changer, et où les prestataires de services comprennent les divers obstacles qui entravent la participation quotidienne au traitement, notamment : la malnutrition due au

\footnotetext{
${ }^{16}$ P. Kopenic, "Interview of Kamil Ivanek, Slovakia, Regional Court of Bratislava", in D. Das and R. Lowe (eds.) Trends in the Judiciary: Interviews with Judges Across the Globe, Volume Two (USA: CRC Press, 2015): 129-150.

17 J. Y. Sacks, S. Sacks, M. Harle et G. D. Leon, "Homelessness prevention therapeutic community (TC) for addicted mothers", Alcoholism Treatment Quarterly 17, no. 1-2 (1999): $33-51$.
} 
manque de revenus, la maladie due au fait de ne pas être traité médicalement, et l'absence de domicile fixe. Ainsi, les consommateurs de drogues problématiques ont besoin de quelqu'un qui les aide vraiment à surmonter leurs difficultés et qui élimine les obstacles à la participation.

Au total, 31 participants ont été interrogés. Sur ces 31, 27 participants venaient du tribunal de district de la juridiction urbaine et rurale (17 au tribunal urbain et 11 au tribunal rural) et trois étaient des juges de la Cour suprême. Cela inclut les juges mutés dans une autre juridiction mais toujours prêts à participer. Sur les 31 participants, 9 étaient des femmes et 22 des hommes. Les entretiens ont été organisés à l'avance et ont duré entre 27 et 97 minutes chacun. Il s'agissait d'une période de travail sur le terrain allant de décembre 2015 à mars 2016. En explorant les perceptions des juges indonésiens concernant les influences sur leur pratique de la condamnation, cette étude fournit des données originales sur la façon dont des questions telles que la pression sociale, les contraintes de temps, le manque de ressources pour le traitement et la persistance des infractions entrent dans les délibérations des juges.

Pour cette étude, les audiences ont été observées toutes les deux semaines par tribunal $(\mathrm{n}=8)$, y compris l'observation des condamnations pour les infractions liées à la drogue. Une liste d'observation de base a été utilisée pour noter les interactions des juges avec les délinquants pendant l'audience de détermination de la peine. Cette liste comprenait des interactions observables telles que:

Dialogue direct entre les juges et les délinquants en cours de condemnation. L'attention des juges sur les discours en matière d'atténuation et la façon dont les juges ont semblé répondre à l'auteur de l'infraction. L'étude a été approuvée par le comité d'éthique de l'université de Stirling et a été financée par le Fonds de dotation indonésien pour l'éducation (LPDP). Chacune des interviews a été enregistrée et ensuite soigneusement transcrite dans son intégralité. Les transcriptions ont ensuite été codées par thème et traduites en anglais. Les efforts déployés pour protéger la confidentialité des informations ont consisté à stocker en toute sécurité les fichiers audio et les documents originaux, et à protéger les transcriptions stockées électroniquement par des mots de passe. Une version anonyme de chaque transcription a été préparée, toutes les informations d'identification étant supprimées. Des procédures de gestion des données minutieuses sont essentielles pour garantir la fiabilité des études qualitatives. ${ }^{18}$

\section{B. Discussion}

\footnotetext{
${ }^{18}$ A. Ashworth, E. Genders, G. Mansfield, J. Peay, et E. Player, Sentencing in the Crown Court (UK: Centre de recherche criminologique de l'Université d'Oxford, 1984)
} 


\section{Perspectives récentes sur les condamnations pour les infractions liées à la drogue en Indonésie Inégalité}

L'inégalité des peines s'inscrit dans une discussion historique plus large sur le droit comme moyen de contrôle des classes inférieures. Les entretiens avec les juges dans le cadre de cette étude ont montré que l'après. La loi indonésienne de 2009 sur les drogues peut être interprétée comme visant les personnes classe. La plupart des personnes accusées d'avoir enfreint cette loi sur les drogues sont issues de milieux défavorisés/pauvres et il semble que le système de justice pénale cible les personnes les plus susceptibles de connaître la pauvreté. Avant la réforme, la consommation de drogue était considérée comme une "tendance". En revanche, à l'époque de la réforme, la consommation de drogue était considérée comme une "tendance". la consommation était considérée comme un crime. (Le terme "réforme" fait référence à la fin de l'ère du Nouvel Ordre sous le régime de Suharto. La loi $39 / 1997$ sur les drogues est née après l'ère de la réforme. Après l'ère de la Réforme, les dispositions relatives à la condamnation pour trafic de drogue ont été fixées dans le cadre des peines minimales).

En 2008, lorsque l'ancienne loi sur les drogues était en vigueur, [il y avait un plus grand nombre de consommateurs de drogues mais un nombre plus faible de personnes condamnées]. [...]. À cette époque, les stupéfiants étaient considérés comme une "tendance". Par exemple, les classes d'élite qui traînaient dans la rue en haut de la colline prenaient souvent de l'ecstasy un dimanche soir. Les personnes qui résidaient en bas de l'échelle considéraient cela comme le style de vie et la consommation de drogue de choix des classes d'élite. Aujourd'hui, la consommation de drogue est considérée comme un crime, et la personne accuséelaccusée de consommation de drogue est en moyenne issue d'un milieu défavorisélpauvre. (Juge 5).

Les préoccupations du juge 5 portaient sur les effets discriminatoires que la guerre contre la drogue avait sur les citoyens des classes inférieures. Les juges participants ont fréquemment expliqué que la majorité des personnes accusées d'avoir enfreint la nouvelle loi sur les drogues étaient issues de milieux sociaux défavorisés. Il semble que le système de justice pénale actuel cible les personnes de classe inférieure. L'extrait suivant indique ce point : "Jusqu'à présent, comme je l'ai observé au cours de la cour, le délinquant que j'ai condamné n'est pas une personne de classe moyenne, donc, en fait, sont rarement de la classe moyenne" (Juge 8). Les entretiens avec les juges participants ont montré que la consommation de drogue était liée à l'impact de la pauvreté. L'affirmation du juge 8 a attiré l'attention sur le fait que la pauvreté influençant le choix d'une personne de vendre de la drogue et lui donnait des drogues gratuites à consommer. Pour comprendre la relation de cause à effet entre la pauvreté et la consommation de drogue, le juge 14 a suggéré les liens suivants: 
En milieu urbain, le chômage devient un problème ; c'est la raison pour laquelle les gens veulent vendre de la drogue, car ils reçoivent une commission non seulement pour la vente de la drogue, mais aussi des drogues gratuites à consommer.

Le contexte de la juridiction urbaine en tant que lieu de tourisme a été considéré par les juges urbains participants comme contribuant aux infractions liées à la drogue.

En ce qui concerne le lien entre la consommation de drogue et la classe inférieure, les participants ont noté trois explications différentes pour expliquer pourquoi les citoyens de la classe inférieure ont le potentiel se livrer à des infractions mineures en matière de drogue. Tout d'abord, les citoyens des classes inférieures étaient enclins à être impliqués dans la culture de la drogue. Cela se reflète dans la déclaration suivante d'un juge rural participant.

Il y a une personne B qui a été persuadée au départ de consommer de la drogue et qui, ensuite, a été contrainte de la distribuer. Lorsqu'il n'avait pas de capital pour acheter de la drogue, qu'il vivait dans une culture de la drogue et qu'il avait besoin d'argent pour survivre, il pouvait alors exercer une double activité : vendre et consommer de la drogue à la commission. (Le juge 19).

Les juges ruraux participants ont considéré que la situation sociale du délinquant était un facteur influençant les infractions mineures en matière de drogue. Dans le même ordre d'idées, les juges urbains participants ont estimé que le chômage avait une influence sur les infractions mineures liées à la drogue. Les deux groupes de juges ont décrit comment le chômage a conduit à des infractions mineures en matière de drogue. Pour les juges des tribunaux urbains, le chômage dans la juridiction rurale a conduit à des infractions mineures en matière de drogue. Pour les juges des tribunaux ruraux, les travailleurs des classes inférieures avaient la double activité de vendre et de consommer de la drogue pour survivre. D'autres participants ont affirmé que le lien entre les citoyens des classes inférieures et les délits mineurs liés à la drogue était dû au fait que les citoyens des classes inférieures consommaient de la drogue pour pouvoir continuer à travailler dur.

J'ai demandé aux délinquants : "Pourquoi utilisez-vous de la Shabu [méthamphétamine] ? 90\% d'entre eux, qui viennent de la classe inférieure, ont répondu : "le premier est de rendre le corps plus fort", c'est la perception dominante chez les délinquants qui consomment de la drogue. $10 \%$ d'entre eux, issus de la classe moyenne, ont répondu que "la drogue est perçue comme un mode de vie". (Juge 28).

Cette proportion importante de citoyens de classe inférieure a indiqué qu'il y avait eu discrimination. En outre, les entretiens avec les juges participants dans les deux juridictions ont montré que la structure de classe contribuait aux infractions liées à la drogue: les drogues semblent être devenues la maladie, parfois, ils n'étaient pas conscients des effets et ils continuent à les utiliser. Ces circonstances me rendent sympathique, parce qu'ils font tout pour se 
procurer de la drogue. Ils vendent tout ce qui est disponible, y compris le vol. (Juge 27).

une fois que la personne est dépendante de la drogue, elle peut faire de l'achat collectif ou d'autres moyens comme le vol. C'est la raison pour laquelle, bien que son travail ne soit que celui d'un chauffeur, il peut faire du Shabu. (Juge 8)

La relation de cause à effet entre le fait d'être pauvre et les infractions liées à la drogue fait écho à d'autres études récentes. Ces études ont suggéré que les citoyens des classes inférieures risquaient d'être arrêtés parce qu'ils avaient tendance à être impliqués dans d'autres infractions afin d'alimenter leur besoin de drogue ; cela les a amenés à devenir une cible de la justice pénale. Au cours des observations du chercheur, les juges urbains participants ont montré qu'ils contestaient le choix du délinquant de dépenser de l'argent.

Jury: Cela aurait été la même valeur d'argent d'acheter du poisson mais trop de poisson vous endort, et j'ai entendu dire que l'utilisation du Shabu vous réveille toute la journée, n'est-ce pas?

Délinquant: C'est vrai;

Panel de juges: Quel est votre travail?

Délinquant: Je vends des produits d'épicerie;

Panel de juges: Le Shabu vous rend donc plus fort pour soulever les courses?

Délinquant: Oui.

La majorité des personnes accusées d'avoir enfreint cette nouvelle loi sur les drogues sont issues de milieux sociaux défavorisés et il semble que l'actuel système de justice pénale indonésien vise les citoyens des classes inférieures. L'extrait suivant indique ce point :

... pour l'instant, les personnes arrêtées sont pour la plupart des "tanggung" (délits de bas niveau), tandis que les trafiquants de drogue restent cachés, et leurs affaires se terminent avec la police. En revanche, ceux qui consomment de l'alcool ou de la drogue, bien que la quantité de drogue ne soit que de zéro virgule zéro, seront traduits en justice. (Juge 12).

Alors que les juges de district participants ont mentionné comment la "classe" décidait souvent si les délinquants mineurs en matière de drogue devaient être punis ou traités, les juges des tribunaux inférieurs participants ont noté que la structure de la classe influençait directement les expériences de condamnation des juges de deux manières différentes. Premièrement, la majorité des participants ont suggéré que la plupart des citoyens des classes inférieures ne recevaient pas de traitement. La déclaration suivante d'un juge de tribunal urbain indique ce point:

Ils [les drogues] ont un double rôle, par exemple, le prix des drogues est de 50000 Rp (environ 2 £), la personne recevra une commission à la fois du vendeur et de l'acheteur et sera autorisée à avoir l'échantillon de drogues pour son propre usage. (Juge 14) 
Le juge de la Cour urbaine a affirmé que les consommateurs de drogues étaient caractérisés par un manque d'emploi stable, ce qui les conduisait à consommer de la drogue. Il semble également que le juge ait reconnu clairement que ces consommateurs de drogue étaient des citoyens de classe inférieure. Cela suggérait que le fait d'être pauvre était à l'origine de l'incapacité à échapper à la culture de la drogue.

Le deuxième impact de la structure des classes sur la détermination de la peine a été perçu par trois participants comme un processus relationnel ayant une incidence sur la détermination de la peine. Plus précisément, il est apparu que la réponse de la Cour suprême à ce sujet faisait écho à la préoccupation judiciaire concernant les citoyens des classes inférieures qui souffrent de l'emprisonnement.

Il a été convenu que l'évaluation est nécessaire pour la réhabilitation. Nous nous appuyons donc sur l'évaluation médicale concernant le niveau de dépendance aux drogues. Si l'exigence d'évaluation n'est pas remplie, même si le délinquant est un consommateur de drogue, il ne recevra pas de réhabilitation. Cela soulève des inquiétudes quant aux délinquants qui ne peuvent pas bénéficier d'une réhabilitation et qui finiront en prison. (Juge 30)

Ces commentaires indiquent explicitement que les délinquants qui ne sont pas en mesure de satisfaire aux exigences de la réadaptation finissent en prison. En revanche, les consommateurs de drogue de la classe moyenne sont cachés du système de justice pénale.

\section{Les défis à relever pour fournir un traitement de la toxicomanie La question des ressources}

Une partie de la difficulté de la condamnation semble provenir du fait que le tribunal n'a pas répondu au besoin de soutien de la majorité des consommateurs de drogue. En expliquant pourquoi il en était ainsi, tous les juges participants ont fait référence au manque de fonds pour payer le transport et le traitement, ce qui rendait difficile l'accès au traitement pour les consommateurs de drogue. Une partie des contraintes pesant sur la condamnation des petits délinquants en matière de drogue semble être imputable le manque d'installations de traitement. Les entretiens avec les juges des tribunaux ruraux participants ont révélé leurs préoccupations quant au fait que les installations de traitement n'étaient disponibles que dans la capitale et non dans tous les districts. L'extrait suivant illustre ce point : "le fait que les installations de traitement ne soient disponibles que dans la capitale et non dans tous les districts a entraîné la surpopulation des prisons" (juge 25). Les juges des tribunaux ruraux participants ont fréquemment exprimé leurs préoccupations quant au manque de soutien disponible pour les consommateurs de drogues. L'extrait suivant illustre ce point:

Nous sommes parfois confrontés à un dilemme dans la condamnation de ces consommateurs de drogue.... Chaque fois, nous avons ordonné au 
procureur de nous aider à faciliter une évaluation médicale. Comme il n'a pas de fonds, le procureur a eu du mal à faire une évaluation et cela devient alors un obstacle. Cela a eu pour conséquence que les délinquants ont été accusés différemment qu'ils ne devraient l'être. Cela a placé le délinquant dans une situation désavantageuse. (Juge 27).

Le fait que le procureur n'ait pas procédé à une évaluation du délinquant a souvent eu pour conséquence que les délinquants ont été inculpés différemment de ce qui aurait dû être le cas et a conduit les juges à ne pas condamner les délinquants à la réhabilitation. En conséquence, les juges des tribunaux ruraux ont considéré que l'absence d'évaluation des délinquants avait un effet négatif sur la détermination de la peine.

Cette compréhension s'est également reflétée dans la réponse des juges des tribunaux urbains participants à ce sujet. Les juges des tribunaux urbains participants étaient conscients que ces tribunaux inférieurs n'avaient pas de possibilités de réinsertion et que cela les empêchait de condamner les délinquants à la réinsertion. L'extrait suivant illustre ce point :

Nous rappelons aux juges de la Cour suprême que "dans une telle juridiction inférieure, il n'y a pas de possibilités de réhabilitation", les juges de la Cour suprême ne condamnaient alors pas les délinquants à la réhabilitation. (Juge 2).

Le juge 2 était conscient que le manque d'installations de traitement faisait partie de la difficulté à condamner les délinquants à la réhabilitation.

\section{L'impact de l'application de la loi}

Une autre partie de la difficulté de la détermination de la peine semble provenir de l'étendue de l'influence du procureur sur l'affaire. Les juges se sentaient contraints par les mises en accusation des procureurs : Nos peines dépendent beaucoup de l'acte d'accusation initial" (juge 7). D'autres se sont inquiétés du fait que les condamnations inférieures au minimum entraînent souvent un appel du procureur.

Je suis très contrarié lorsqu'il y a un cas où le contrevenant pourrait être inculpé en vertu de l'article 127 en raison de la plus petite quantité de drogue, mais qu'il n'est pas inculpé en vertu de l'article 127. En revanche, lorsque la quantité de preuves est plus importante, le contrevenant est inculpé en vertu de la règle 127... (Juge 23)

Les observations du chercheur sur le tribunal rural ont révélé que le procureur a consulté le panel de juges. Cela indique l'influence du procureur sur les décisions finales en la matière.

Comité des juges : ...Nous faisons une pause maintenant! ...

Panel de juges : Ok, la séance continue [première phase de la condamnation], nous décidons de réduire la peine de 5 à 4 ans, c'est le minimum. Comment vous sentez-vous?

Délinquant : [pleuré]. 
La citation ci-dessus souligne la préoccupation du juge 23 selon laquelle, pour les petites quantités de drogue, le procureur a souvent engagé des poursuites sévères alors que, pour les grandes quantités de drogue, le procureur a souvent engagé des poursuites indulgentes. Il est évident que la réponse de la Cour suprême à ce sujet fait écho à l'explication donnée sur le fait que l'inculpation initiale est une contrainte pour la détermination de la peine.

Nous comprenons à peine ce qui s'est passé derrière l'acte d'accusation... il a été constaté après l'audience du tribunal que le délinquant avait été condamné en tant que consommateur de drogue; cependant, la règle 127 sur les consommateurs de drogue ne faisait pas partie de l'acte d'accusation. Par conséquent, cela posait un problème si nous appliquons la loi de manière stricte et si les preuves montraient que le délinquant était un consommateur de drogue, alors que la règle sur la consommation de drogue ne faisait pas partie de l'acte d'accusation. (Juge 30).

Cette constatation suggère que les contradictions entre les actes d'accusation déposés et les preuves factuelles révélées au tribunal ont eu un impact négatif sur la détermination de la peine. L'extrait suivant montre que les juges se sont souvent sentis contraints par la procédure d'appel lorsqu'ils ont condamné en dessous de la peine minimale:

Je suis conscient que si l'auteur de l'infraction est condamné à une peine inférieure au minimum, il fera certainement l'objet d'un appel. En outre, cela entraînera des conséquences inattendues qui rendront les choses plus difficiles pour le délinquant. Ces pratiques sont devenues une habitude, cela arrive souvent. (Juge 5).

Le juge 5 a expliqué que l'appel du ministère public avait souvent des conséquences imprévues qui rendaient les choses plus difficiles pour l'auteur de l'infraction. Ce faisant, la procédure d'appel a été considérée comme ayant un impact négatif sur le délinquant.

\section{Réhabilitation}

Les juges ont largement accepté la réhabilitation comme objectif de la condamnation, en particulier pour les jeunes toxicomanes et les délinquants primaires. Voir par exemple ces deux commentaires:

Cet objectif [de réhabilitation] serait préférable à leur incarcération car, une fois qu'ils seront en contact avec d'autres dealers et consommateurs de drogue à l'intérieur de la prison, cela aura un impact négatif sur leur santé mentale. (Juge 17).

Le fait est que nous devons être honnêtes, en milieu rural, et généralement aussi dans d'autres endroits. La punition, en fait, n'a pas résolu le problème. Nous constatons qu'après l'entrée en prison du consommateur de drogue, elles seront plus sévères. (Juge 26) 
La réhabilitation a été considérée comme appropriée plutôt que l'emprisonnement, ce qui n'est pas déraisonnable. Mais la réhabilitation est également considérée comme étant dans l'intérêt de la société dans son ensemble:

Ces délinquants, dont les familles sont victimes de la consommation de drogue, s'attendent à une réhabilitation..... Cela correspond peut-être à l'attente de la société qui veut que les installations de traitement soient adéquates. (Juge 27).

Plusieurs autres juges (dont un qui a déclaré que la prison ne réhabilite pas) ont également déclaré qu'elle pouvait, dans certaines circonstances, avoir un effet de réhabilitation. Ce à quoi ils font probablement référence ici, c'est à la dissuasion individuelle.

Je pense que la fonction de la prison est essentiellement celle d'un établissement pénitentiaire, cela s'applique à tous les cas. Donc, en fait, tant que le délinquant en centre de détention peut être guéri, alors il n'est pas nécessaire de le mettre en prison. (Juge 19).

J'ai eu des nouvelles de la belle-sœur du délinquant, qui travaillait chez moi, et je me suis rendu compte que le délinquant avait changé après sa condamnation. Auparavant, le délinquant vendait et consommait de la drogue ; aujourd'hui, après sa sortie de prison, il ne consomme plus de drogue. (Juge 22).

Les juges de la Cour suprême participants ont souligné que la politique visant à mettre fin aux sanctions exigerait que la police et l'Agence nationale des stupéfiants (BNN) modifient leurs pratiques sur le terrain. En effet, au stade de l'enquête, la décision de détourner les consommateurs de drogue est entre les mains de la police et de la BNN.

Il semble possible, si le consommateur de drogue est déja au début le consommateur de drogue n'entrera plus dans le tribuna. De notre point de vue, il serait bon que le consommateur de drogue puisse être réhabilité et non pas puni parce que inévitablement, cela nous aide aussi. (Juge 30).

L'extrait ci-dessus met en évidence l'attente du juge de la Cour suprême selon laquelle l'initiative de la déjudiciarisation partirait de la base lors de la phase d'enquête. Ce faisant, la volonté de la police de modifier sa pratique au début aurait un impact sur la réhabilitation des mineurs délinquants toxicomanes.

\section{Conclusion}

Ces résultats sont en accord avec l'idée que l'impact de la pauvreté de classe sur la consommation de drogue peut être mesuré et compris comme un processus relationnel qui détermine les délits mineurs liés à la drogue. On peut donc affirmer que la consommation de drogue en Indonésie reflète l'inégalité économique dans des structures sociales plus larges, à la réalité de la société qui est elle-même injuste. Dans l'ensemble, les problèmes structurels liés à la 
consommation de drogues semblent s'écarter de la recherche contemporaine sur le traitement des toxicomanes, qui a préconisé la nécessité pour les consommateurs de drogues d'avoir accès à un emploi stable. ${ }^{19}$

De plus, en gardant à l'esprit que la plupart des personnes amenées devant le tribunal étaient des citoyens de classe inférieure, les participants ont affirmé que le lien entre les citoyens de classe inférieure et le ciblage des drogues concordait avec la notion de guerre contre les drogues et que le système de justice pénale avait tendance à cibler les consommateurs de drogues qui étaient des citoyens de classe inférieure. ${ }^{20}$

Cela suggère que la "classe" décidait souvent si les petits délinquants toxicomanes devaient être punis ou traités. Les juges des tribunaux inférieurs participants ont noté que la structure des classes avait une influence directe sur la condamnation de deux manières différentes. Premièrement, la plupart des toxicomanes des classes inférieures ne recevaient pas de traitement. Deuxièmement, la guerre contre la drogue visait les citoyens des classes inférieures et était discriminatoire. Cela est dû au fait que la majorité des personnes accusées d'avoir enfreint la loi sur les drogues après 2009 étaient issues de milieux sociaux défavorisés. En revanche, les consommateurs de drogue de la classe moyenne ont échappé au système de justice pénale et n'ont pas été la cible de ses sanctions.

Cette étude qualitative suggère également qu'une partie de la difficulté à faire usage des dispositions de traitement provient du fait que les juges se sentent contraints par les mises en accusation des procureurs, la procédure d'appel, les évaluations médicales et la disponibilité des installations de traitement. Néanmoins, les juges ont estimé que la réhabilitation des mineurs délinquants toxicomanes pouvait à la fois faciliter leur rétablissement et réduire la charge de travail des tribunaux.

\section{References}

Iskander, I. "Decriminalisation to Drug User are not similar with Legalisation". (2014) Disponible à l'adresse suivante: http://anangiskandar.wordpress.com/ [consulté le 6 octobre 2014].

Ashworth, A., Genders, E., Mansfield, G., Peay, J., et Player, E. Sentencing in the Crown Court. Centre de recherche criminologique de l'Université d'Oxford, 1984.

Ashworth, A. Sentencing and Criminal Justice. Cambridge : Université de Cambridge Presse, 2010.

\footnotetext{
19 J. Buchanan, "Understanding Problematic Drug Use: une question médicale ou sociale Issue", British Journal of Community Justice 4, no. 2 (2006): 387-397.

${ }^{20}$ E. A. Nadelmann, "Criminologist and Punitive Drug Prohibition: to serve or to challenge?" Criminologie et politique publique 3, no. 3 (2004): 441-450. https://doi.org/10.1111/j.17459133.2004.tb00052.x.
} 
BNN Data Analysis of Drug Prevention and Enforcement 2013 (Jakarta: Indonesian Narcotics Board, 2013. [en ligne] Disponible sur : http://103.3.70.3/portal/_uploads/post/2014/09/02/Analisa_Data_P4G N_Tahun_2013.pdf [Accédé le 23 novembre 2014].

Buchanan, J. "Ending Drug Prohibition with a Hangover", British Journal of Justice communautaire 13, no. 1 (2015): 55-74.

Buchanan, J. "Understanding Problematic Drug Use : une question médicale ou sociale Issue", British Journal of Community Justice 4, no. 2 (2006): 387-397.

Buchanan, J. "Tackling Problem Drug Use : A New Conceptual Framework", Social Work in Mental Health 2, no. 2-3 (2004): 117-138.

D. Garland and R. Duff (eds.) A Reader on Punishment. Oxford : Oxford University Press, 1994.

Chambliss, W. J. "Toward a Political Economy of Crime", Theory and Society 2, no. 1(1975): 149-170.

Nomura, K. "Et la justice pour tous? Racial and Ethnic Disparities in Federal Drug Courts in California and the US", California Journal of Politics and Policy 7, no. 2 (2015).

Duff, R.A. et Garland, D. A Reader on Punishment. Oxford : Université d'Oxford Presse, 1994.

Garland, D. The Culture of Control: Crime et ordre social dans la société contemporaine. USA: University of Chicago Press, 2001.

Gibson, J. L. "From simplicity to complexity : The development of theory in the study of judicial behaviour". Political Behaviour 5, no. 1(1983): 749.

Holmes, O.W. The Common Law. Harvard University Press, 2009.

Hutton, N. "Sentencing as a Social Practice", dans S. Armstrong et L. McAra (eds.). Perspectives on Punishment : the Contours of Control. Oxford: Oxford University Press, 2006.

Kopenic, P "Interview of Kamil Ivanek, Slovakia, Regional Court of Bratislava", in D. Das and R. Lowe (eds.) Trends in the Judiciary : Interviews with Judges Across the Globe, Volume Two. France: CRC Press, 2015.

Lassiter, M. D. "Impossible Criminels: The Suburban Imperatives of America's War on Drugs", Journal of American History 102, no. 1 (2015): 126-140.

Loi 35/(2009) (loi indonésienne sur les stupéfiants)

McSweeney, T., Turnbull, P.J., et Hough, M. The Treatment and Supervision of Drug-Dependent Offenders : A Review of the Literature Prepared for the UK Drug Policy Commission. Londres : Institute for Criminal Policy Research, King's College London, 2008.

Melossi, D. Controlling Crime, Controlling Society : Thinking about Crime in Europe and America. Polity, 2008. 
Mulyadi. Punition contre les trafiquants et les consommateurs de drogues : Research on Principles, Theory, and Practice Norms Application in Court. Jakarta: Centre de recherche pour la Cour suprême indonésienne, 2012.

Myers, M.A. et Talarico, S. M. The Social Contexts of Criminal Sentencing. Springer Science et médias d'affaires, 1987.

Nadelmann, E. A. "Criminologist and Punitive Drug Prohibition : to serve or to challenge?" Criminologie et politique publique 3, no. 3 (2004): 441450. https://doi.org/10.1111/j.1745-9133.2004.tb00052.x.

Nunn, K. B. "Race, crime and the pool of surplus criminality : or why the war on drugs was a war on blacks", Gender Race and Justice 6, no. 381 (2002).

Pettit, B. et Western, B. "Mass imprisonment and the life course: Race and class inequality in US incarceration", American Sociological Review 69, no. 20 (2004): https://doi.org/10.1177/000312240406900201.

Provine, D. M. "Race et inégalité dans la guerre contre la drogue", Revue annuelle du droit et de la politique de l'Union européenne Social Science 7 (2011): 41-60.

Quinney, R. Critique de l'ordre juridique: Le contrôle du crime dans la société capitaliste.USA: Transaction Publishers, 1974.

Rusche, G. et Kirchheimer, O. Punishment and Social Structure. USA: Transaction Éditeurs, 2003.

Sacks, J. Y., Sacks, S., Harle, M. et Leon, G.D. "Homelessness prevention therapeutic community (TC) for addicted mothers", Alcoholism Treatment Quarterly 17, no. 1-2 (1999): 33-51.

Shiner, M. "Drug policy reform and the reclassification of cannabis in England and Le Pays de Galles: A cautionary tale", International Journal of Drug Policy 26, no. 7: 696-704 (2015). https://doi.org/10.1016/j.drugpo.2015.03.009.

Spohn, C. "Evolution of Sentencing Research". Criminology and Public Policy 14, no. 2 (2015): 225-232.

Stevens, A. "Quasi-compulsory treatment in Europe : an evidence-based response to drug related crime" dans A. Stevens (ed.) Crossing Frontiers : International Developments in the Treatment of Drug Dependence. UK: Pavillon, 2008.

Stevens, A., Berto, D., Frick, U., Hunt, N., Kerschl, V., McSweeney, T., Oeuvray, K., Puppo, I., Santa Maria, A., Schaaf, S., Trinkl, B., Uchtenhagen, A., et Werdenich, W. "The relationship between legal status, perceived pressure and motivation in treatment for drug dependence: results from a European study of Quasi-Compulsory Treatment". European Addiction Research 12, no. 4 (2006): 197-209. 
Service pénitentiaire indonésien Rapport annuel (Jakarta : Ministère de la justice et des affaires humaines Rights) [en ligne] (2013) Disponible à l'adresse suivante http://www.ditjenpas.go.id/sites/default/files/ANUAL\%20REPORT\% 20REVISI\%20TERAKHIR\%20hq\%20print.pdf [consulté le 8 février 2015].

Ulmer, J. T. Social Worlds of Sentencing: Communautés judiciaires sous le coup d'une condamnation Lignes directrices. USA: SUNY Press, 1997.

Vanhamme, F. and Beyens, K. "La recherche end sentencing: un survol contextualize", Déviance et Société 31, no. 2 (2007): 199-228. DOI: 10.3917/ds.312.0199.

Ward, J. "Punishing Drug Possession in the Magistrates' Courts: Il est temps de Rethink", European Journal on Criminal Policy and Research 19, no. 4 (2013): 289-307. 
